\title{
Total synthesis of amidepsine $B$ and revision of its absolute configuration
}

\author{
Tohru Nagamitsu ${ }^{1}$, Kaori Marumoto ${ }^{1}$, Asami Nagayasu${ }^{1}$, Takeo Fukuda ${ }^{1}$, Shiho Arima ${ }^{1}$, Ryuji Uchida ${ }^{1}$, \\ Taichi Ohshiro ${ }^{1}$, Yoshihiro Harigaya ${ }^{1}$, Hiroshi Tomoda ${ }^{1}$ and Satoshi Ōmura ${ }^{2}$
}

The total synthesis of (-)-amidepsine B, a potent diacylglycerol acyltransferase inhibitor, has been achieved. This synthetic study resulted in the revision of the previously assigned stereostructure of the natural amidepsine $B$ and determined the absolute configuration.

The Journal of Antibiotics (2009) 62, 69-74; doi:10.1038/ja.2008.12; published online 23 January 2009

Keywords: absolute configuration; (-)-amidepsine B; total synthesis

\section{INTRODUCTION}

Amidepsines A-E were isolated from the culture broth of fungal strains FO-2942 and FO-5969 and found to be inhibitors of diacylglycerol acyltransferase (DGAT), ${ }^{1-3}$ which is exclusively involved in triacylglycerol formation. Excessive accumulation of triacylglycerol can cause fatty liver, obesity and hypertriglyceridemia, which leads to serious diseases, such as atherosclerosis, diabetes and metabolic disorders. Therefore, DGAT inhibitors have the potential to become drugs. Spectroscopic analyses of the amidepsines elucidated depsipeptide structures consisting of three 4,6-dihydroxy-2-methylbenzoic acid derivatives and an amino acid (except for amidepsine D), as shown in Figure 1. The DGAT inhibitory activity of the amidepsines was tested by a cellular assay using Raji cells, and the results showed that amidepsine B (1) was the most potent inhibitor. Amidepsine B (1) was previously determined to be a mixture of stereoisomers at its single chiral center, the alanine alpha carbon. A 3:2 mixture of L- and D-alanines was revealed by acid hydrolysis followed by HPLC analyses using a chiral column (Amidepsines $\mathrm{A}$ and $\mathrm{C}$ were also reported as a 3:2 mixture of L- and D-amino acids ${ }^{2}$ ). Herein, the total synthesis of amidepsine $\mathrm{B}$ and a revision of its absolute configuration will be described.

\section{RESULTS AND DISCUSSION}

The synthesis used a known aldehyde 2 , which is a common starting material for the preparation of key substrates $\mathbf{5}$ and $\mathbf{1 0}$ (scheme 1). ${ }^{4}$ Regioselective $p$-O-methylation (In this reaction, reactivity of the hydroxy group adjacent to the formyl group in $\mathbf{3}$ would be precluded by the formation of an intramolecular hydrogen bond. Similar reactivity was observed. ${ }^{5}$ ) followed by benzylation gave 4 quantitatively, which was treated with $\mathrm{NaClO}_{2}$ to afford carboxylic acid $\mathbf{5}$ quantitatively. Furthermore, similar conversion of $\mathbf{2}$ to $\mathbf{8}$, including the formation of a $p$-O-methoxymethyl ether instead of methylation, was also accomplished quantitatively. Esterification of $\mathbf{8}$ with allyl bromide followed by deprotection of the methoxymethyl ether afforded $\mathbf{1 0}$ quantitatively.

Next, the key substrates $\mathbf{5}$ and $\mathbf{1 0}$ were coupled by treatment with trifluoroacetic anhydride (TFAA) to give rise to 11 quantitatively. Deprotection of the allyl ester in the presence of a palladium catalyst gave carboxylic acid $\mathbf{1 2}$ in $98 \%$ yield.

Subsequent esterification of $\mathbf{1 2}$ with $\mathbf{1 0}$ was troublesome. Esterification conditions, such as TFAA, 1-[(3-dimethylamino)propyl]-3-ethylcarbodiimide/dimethylaminopyridine (DMAP) and benzotriazol-1yloxytris(dimethylamino)phosphonium hexafluorophosphate (BOP)/ $\mathrm{Et}_{3} \mathrm{~N} / \mathrm{DMAP}$ led to a low yield of the desired product 14 with side products, such as the anhydride of $\mathbf{1 2}$. Therefore, we next focused on esterification through the acid fluoride. ${ }^{6}$ Reaction of $\mathbf{1 2}$ with (diethylamino)sulfur trifluoride (DAST) proceeded in $89 \%$ yield to furnish the stable acid fluoride $\mathbf{1 3}$. Treatment of $\mathbf{1 3}$ with sodium alkoxide, derived from 10 and sodium hydride, was effected to give the desired allyl ester $\mathbf{1 4}$ quantitatively. The allyl ester was then deprotected under palladium-catalyzed conditions to afford $\mathbf{1 5}$ quantitatively.

Having constructed the depside 15, we next turned to condensation with L-alanine before preparing the 3:2 mixture of $\mathrm{L}$ - and D-alanines toward the total synthesis (scheme 2). Treatment of $\mathbf{1 5}$ with L-alanine benzyl ester in the presence of BOP and $\mathrm{Et}_{3} \mathrm{~N}$ produced benzyl ester (+)-16 in 79\% yield. Finally, global deprotection by hydrogenolysis completed the total synthesis of (-)-amidepsine B $[(-)-\mathbf{1}]$ in $83 \%$ yield.

The physical properties $\left({ }^{1} \mathrm{H}\right.$ and ${ }^{13} \mathrm{C}$ NMR, m.p., IR and MS) of synthetic (-)-amidepsine B (1) were completely identical to those of a

${ }^{1}$ School of Pharmacy, Kitasato University, Tokyo, Japan and ${ }^{2}$ Kitasato Institute for Life Sciences and Graduate School of Infection Control Sciences, Kitasato University, Tokyo, Japan

Correspondence: Professor S Ōmura, Kitasato Institute for Life Sciences and Graduate School of Infection Control Sciences, Kitasato University, 5-9-1 Shirokane, Minato-ku, Tokyo 108-8641, Japan. 
natural sample, and the optical rotation of synthetic $(-)-1\left([\alpha]_{\mathrm{D}}^{23}-\right.$ $\left.17.3^{\circ} ; c 0.10, \mathrm{MeOH}\right)$ also corresponded with that of the authentic sample of $(\mathbf{1})\left([\alpha]_{\mathrm{D}}^{25}-16^{\circ} ; c 0.1, \mathrm{MeOH}\right)$. This result triggered the need for the re-assignment of the absolute configuration of natural<smiles>COc1cc(C)c(C(=O)Oc2cc(C)c(C(=O)Oc3cc(C)c(C(=O)O)c(O)c3)c(O)c2)c(O)c1</smiles>

\begin{tabular}{|cccccc|}
\hline Amidepsine & A & B & C & D & E \\
\hline $\mathrm{R}_{1}$ & $\mathrm{Me}$ & $\mathrm{H}$ & $\mathrm{H}$ & $\mathrm{Me}$ & $\mathrm{Me}$ \\
$\mathrm{R}_{2}$ & $\mathrm{H}$ & $\mathrm{H}$ & $\mathrm{H}$ & $\mathrm{H}$ & $\mathrm{Me}$ \\
$\mathrm{R}_{3}$ & alanine $^{\star \star}$ & L-alanine $^{\star}$ & valine $^{* \star}$ & $\mathrm{OH}$ & alanine \\
\hline
\end{tabular}

Figure 1 Structure of amidepsines $\mathrm{A}-\mathrm{E}$. ${ }^{*}$ The original isomeric mixture reported for amidepsine $\mathrm{B}$ has been corrected by this work. ${ }^{*}$ The actual configurations of amidepsines $\mathrm{A}$ and $\mathrm{C}$ are now in question. ${ }^{2}$ amidepsine B (1). According to the procedure shown above, (+)-amidepsine B [(+)-1] by condensation of $\mathbf{1 5}$ with D-alanine was also synthesized.

We next analyzed the synthetic amidepsines (+)-1 and (-)-1 and the natural amidepsine B (1) with a chiral HPLC column. When the 1:1 mixture of the synthetic amidepsines (+)-1 and (-)-1 was injected onto the chiral HPLC system, each enantiomer was separated completely as shown in Figure $2 \mathrm{a}$. Figures $2 \mathrm{~b}$ and $\mathrm{c}$ show the HPLC analytical data of $(+)-\mathbf{1}$ and $(-)-\mathbf{1}$, respectively. Next, the natural amidepsine B (1) was also subjected to HPLC analysis and it was determined that the retention time was identical to that of $(-)$ amidepsine B [(-)-1] as shown in Figure 2d.

This result shows that the absolute configuration of amidepsine $B$ is $S$, derived from $\mathrm{L}$-alanine, and that the previous determination of the absolute configuration of amidepsine B was inaccurate. It is likely that the acid hydrolysis of amidepsine $\mathrm{B}$ must have led to the epimerization of the resulting alanine. It is highly likely that amidepsines $\mathrm{A}$ and $\mathrm{C}$ are also L-amino acids, which will be reported in due course.

In conclusion, we have achieved the total synthesis of amidepsine B. Moreover, we have revised the absolute configuration of natural amidepsine B. Extension of this chemistry to the synthesis of structural analogs of amidepsine B so that the structure-activity<smiles>COc1cc(C)c(C(=O)OCc2ccccc2)c(C(C)C)c1</smiles><smiles>CCOc1cc(OC)c(C(=O)OCC(C)C)c(OCC(C)C)c1</smiles>

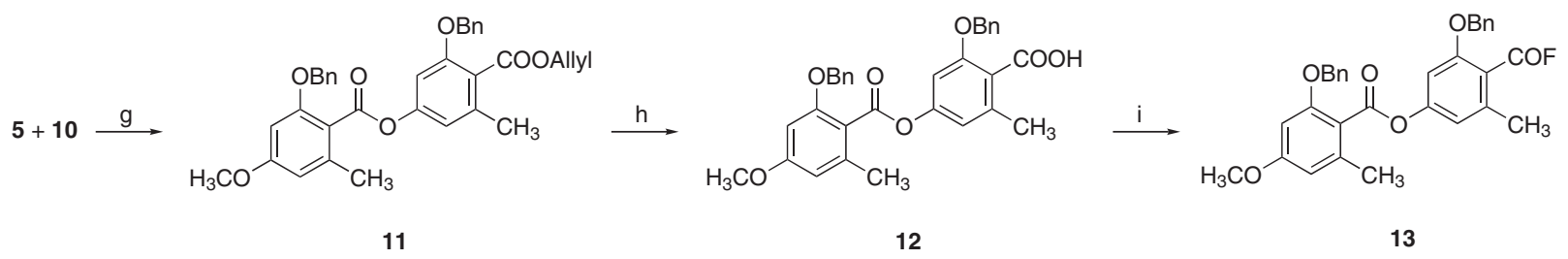<smiles>COc1cc(C)c(C(=O)Oc2cc(C)c(C(=O)Oc3cc(C)c(C(=O)O[Na])c(O)c3)c(O)c2)c(O)c1</smiles><smiles>CCCOc1cc(OC(=O)c2c(C)cc(OC(=O)c3c(C)cc(OC)cc3C)cc2OCC)cc(C)c1C(=O)OCc1ccccc1</smiles>

Scheme 1 Synthesis of depside 15. Reagents and conditions: (a) $\mathrm{CH}_{3} \mathrm{l}, \mathrm{K}_{2} \mathrm{CO}_{3}$, acetone, rt, quant.; (b) Benzyl chloride, $\mathrm{K}_{2} \mathrm{CO}_{3}$, DMF, rt, quant. for 4 and 7; (c) $\mathrm{NaClO}_{2}$, 2-methyl-2-butene, $\mathrm{NaH}_{2} \mathrm{PO}_{4} \cdot 2 \mathrm{H}_{2} \mathrm{O}, t-\mathrm{BuOH} / \mathrm{H}_{2} \mathrm{O}, 0^{\circ} \mathrm{C}$ to rt, quant. for 5 and 8; (d) Methoxymethyl chloride, $i-\mathrm{Pr}_{2} \mathrm{NEt}, \mathrm{CH}_{2} \mathrm{Cl}{ }_{2}, 0^{\circ} \mathrm{C}$ to rt, quant.; (e) Allyl Br, $\mathrm{NaH}, \mathrm{DMF}, \mathrm{O}^{\circ} \mathrm{C}$ to rt, quant.; (f) $6 \mathrm{~N} \mathrm{HCl}, \mathrm{MeOH}, 60^{\circ} \mathrm{C}$, quant.; (g) Trifluoroacetic anhydride, toluene, rt, quant.; (h) $\mathrm{HCOOH}, \mathrm{Pd}\left(\mathrm{PPh}_{3}\right)_{4}, \mathrm{Et}_{3} \mathrm{~N}$, $\mathrm{THF}, \mathrm{O}^{\circ} \mathrm{C}$ to rt, $98 \%$ for 12 , quant. for 15 ; (i) (Diethylamino)sulfur trifluoride, $\mathrm{Et}_{2} \mathrm{O} / \mathrm{CH}_{2} \mathrm{Cl}_{2}$, rt, $89 \%$; (j) $10, \mathrm{NaH}, \mathrm{THF}, 0^{\circ} \mathrm{C}$, then 13 , rt, quant. 
<smiles>CCCCc1cc(OC)cc(C)c1C(=O)Oc1cc(C)c(C(=O)Oc2cc(C)c(C(=O)O)c(OCCC)c2)c(OCC)c1</smiles>

15<smiles>CCCCCOc1cc(OC(=O)c2c(C)cc(OC(=O)c3c(C)cc(OC)cc3C)cc2C(=O)OCc2ccccc2)cc(C)c1C(=O)NC(C)C(=O)OCc1ccccc1</smiles>

$(+)-16$<smiles>COc1cc(C)c(C(=O)Oc2cc(C)c(C(=O)Oc3cc(C)c(C(=O)N[C@@H](C)C(=O)O)c(O)c3)c(O)c2)c(O)c1</smiles>

(-)-Amidepsine B (1)

Scheme 2 Completion of the total synthesis of (-)-amidepsine B [(-)-1]. Reagents and conditions: (k) L-alanine benzyl ester, BOP, Et 3 N, DMF, rt, 79\%. For (-)-16: D-alanine benzyl ester, BOP, $\mathrm{Et}_{3} \mathrm{~N}, \mathrm{DMF}, \mathrm{rt}, 89 \%$; (I) $\mathrm{H}_{2}$, $\mathrm{Pd}(\mathrm{OH})_{2}$, THF/EtOH, rt, 83\%. For (+)-1: $94 \%$.

relationships can be explored is currently under way and will be reported in due course.

\section{EXPERIMENTAL PROCEDURE}

Commercially available solvents were dried and distilled before use. Reactions were monitored by TLC using Merck $\mathrm{F}_{60} 0_{254}$ silica gel plates $(0.25 \mathrm{~mm})$. Spots were visualized with ultraviolet (UV) light $(254 \mathrm{~nm})$ and stained with phosphomolybdic acid. Flash column chromatography was performed on silica gel $60 \mathrm{~N}$ (spherical, neutral, particle size $40-50 \mu \mathrm{m}$ ). Preparative TLC was performed on Merck $\mathrm{F} 60_{254}$ silica gel plates $(0.50 \mathrm{~mm})$. The chiral HPLC system was comprised of a Daicel-CHIRALPAK IA column $(0.46 \mathrm{~cm} \phi \times 25 \mathrm{~cm})$, and a mobile phase of hexane/THF (tetrahydrofuran)/ TFAA (80:20:0.1) (Senshu HPLC system; $10 \mu \mathrm{l}$ injection; UV, $254 \mathrm{~nm}$; flow rate, $\left.1.0 \mathrm{ml} \mathrm{min}^{-1}\right)$.

Melting points were determined on a Yanagimoto micro melting apparatus and uncorrected. Optical rotations were obtained with a JASCO DIP-1000 polarimeter. Mass spectra were recorded on a JEOL JMS-700 mass spectrometer. FT-IR spectra were recorded on a Horiba FT-710 spectrometer. ${ }^{1} \mathrm{H}-$ NMR spectra were recorded on a JEOL JNM-EX270 (270 MHz), MERCURY$300(300 \mathrm{MHz})$ and UNITY-400 $(400 \mathrm{MHz})$ spectrometers in $\mathrm{CDCl}_{3}$ or DMSO$\mathrm{d}_{6} .{ }^{1} \mathrm{H}$-NMR spectral data are reported according to the following conventions: chemical shifts relative to $\mathrm{CDCl}_{3}$ (7.26 p.p.m.) or $\mathrm{DMSO}_{6}$ (2.48 p.p.m.), multiplicity ( $\mathrm{s}=$ singlet, $\mathrm{d}=$ doublet, $\mathrm{t}=$ triplet, $\mathrm{q}=$ quartet, $\mathrm{m}=$ multiplet, $\mathrm{br}$ $\mathrm{s}=$ broad singlet, br $\mathrm{d}=$ broad doublet and $\mathrm{dd}=$ double doublet), coupling constant and integration. ${ }^{13} \mathrm{C}-\mathrm{NMR}$ spectral data are reported in p.p.m. relative to $\mathrm{CDCl}_{3}$ (77.0 p.p.m.) or DMSO-d $\mathrm{d}_{6}$ (39.5 p.p.m.).

\section{2-Hydroxy-4-methoxy-6-methylbenzaldehyde (3)}

To a solution of 2,4-dihydroxy-6-methylbenzaldehyde (2) $(0.56 \mathrm{~g}, 3.68 \mathrm{mmol})$ in acetone $(36.8 \mathrm{ml}), \mathrm{K}_{2} \mathrm{CO}_{3}(0.51 \mathrm{~g}, 3.68 \mathrm{mmol})$ and $\mathrm{CH}_{3} \mathrm{I}(3.44 \mathrm{ml}$, $55.2 \mathrm{mmol}$ ) were added. The reaction mixture was stirred for $2.5 \mathrm{~h}$ at room temperature, quenched with $\mathrm{H}_{2} \mathrm{O}$ and extracted with EtOAc. The combined organic extracts were dried over anhydrous $\mathrm{Na}_{2} \mathrm{SO}_{4}$ and concentrated in vacuo. The residue was purified by flash column chromatography on silica gel with an eluent (20:1 hexanes/EtOAc) to give 3 ( $0.61 \mathrm{~g}$, quant.) as a white powder; m.p. 65-67 ${ }^{\circ} \mathrm{C}$; IR (KBr): 3084, 2966, 2906, 2846, 1635, 1576, 1473 and $1429 \mathrm{~cm}^{-1}$; ${ }^{1} \mathrm{H}-\mathrm{NMR}\left(300 \mathrm{MHz}, \mathrm{CDCl}_{3}\right) \delta 12.45(\mathrm{~s}, 1 \mathrm{H}), 10.05(\mathrm{~s}, 1 \mathrm{H}), 6.23(\mathrm{~s}, 2 \mathrm{H}), 3.80$ (s, 3H), 2.49 (s, 3H); ${ }^{13} \mathrm{C}-\mathrm{NMR}\left(75 \mathrm{MHz}, \mathrm{CDCl}_{3}\right) \delta$ 192.8, 166.6, 166.4, 143.7, 113.2, 110.4, 98.5, 55.4 and 18.2; HRMS (FAB, $m-\mathrm{NBA}$ ): calcd. for $\mathrm{C}_{9} \mathrm{H}_{11} \mathrm{O}_{3}$ : $167.0708[\mathrm{M}+\mathrm{H}]^{+}$, found: $m / z$ 167.0703.
2-Benzyloxy-4-methoxy-6-methylbenzaldehyde (4)

To a solution of $3(0.60 \mathrm{~g}, 3.64 \mathrm{mmol})$ in $\mathrm{N}, \mathrm{N}$-dimethylformamide (DMF) $(18.2 \mathrm{ml}), \mathrm{K}_{2} \mathrm{CO}_{3}(5.03 \mathrm{~g}, 36.4 \mathrm{mmol})$ and $\mathrm{BnCl}(6.29 \mathrm{ml}, 56.4 \mathrm{mmol})$ were added at $0{ }^{\circ} \mathrm{C}$. After stirring for $2.5 \mathrm{~h}$ at room temperature, the reaction was quenched with $\mathrm{H}_{2} \mathrm{O}$ and extracted with $\mathrm{CH}_{2} \mathrm{Cl}_{2}$. The combined organic extracts were dried over anhydrous $\mathrm{Na}_{2} \mathrm{SO}_{4}$ and concentrated in vacuo. The residue was purified by flash column chromatography on silica gel with an eluent (20:1 to 10:1 hexanes/EtOAc) to give $4(0.93 \mathrm{~g}$, quant.) as a white powder; m.p. 78 $80^{\circ} \mathrm{C}$; IR (KBr): 3321, 3091, 3049, 2966, 2925, 2871, 2789, 1666, 1603 and $1448 \mathrm{~cm}^{-1}$; ${ }^{1} \mathrm{H}-\mathrm{NMR}\left(270 \mathrm{MHz}, \mathrm{CDCl}_{3}\right) \delta 10.59(\mathrm{~s}, 1 \mathrm{H}), 7.38(\mathrm{~m}, 5 \mathrm{H}), 6.40(\mathrm{~d}$, $J=1.8 \mathrm{~Hz}, 1 \mathrm{H}), 6.34(\mathrm{~d}, J=1.8 \mathrm{~Hz}, 1 \mathrm{H}), 5.14(\mathrm{~s}, 2 \mathrm{H}), 3.84(\mathrm{~s}, 3 \mathrm{H}), 2.60(\mathrm{~s}, 3 \mathrm{H})$; ${ }^{13} \mathrm{C}-\mathrm{NMR}\left(75 \mathrm{MHz}, \mathrm{CDCl}_{3}\right) \delta 190.4,164.2,164.2,144.6,136.0,128.6,128.1$, 127.2, 117.5, 109.1, 97.0, 70.5, 55.3 and 22.3; HRMS (FAB, $m$-NBA): calcd. for $\mathrm{C}_{16} \mathrm{H}_{17} \mathrm{O}_{3}: 257.1178[\mathrm{M}+\mathrm{H}]^{+}$, found: $m / z$ 257.1176.

\section{2-Benzyloxy-4-methoxy-6-methylbenzoic acid (5)}

To a solution of $4(0.16 \mathrm{~g}, 0.61 \mathrm{mmol})$ in $t-\mathrm{BuOH} / \mathrm{H}_{2} \mathrm{O}(1: 1)(6.2 \mathrm{ml}) 2$-methyl2-butene $(0.26 \mathrm{ml}, 2.45 \mathrm{mmol}), \mathrm{NaH}_{2} \mathrm{PO}_{4} \cdot 2 \mathrm{H}_{2} \mathrm{O}(0.29 \mathrm{~g}, 1.84 \mathrm{mmol})$ and $\mathrm{NaClO}_{2}(0.17 \mathrm{ml}, 1.84 \mathrm{mmol})$ were added at $0{ }^{\circ} \mathrm{C}$. The reaction mixture was stirred for $24 \mathrm{~h}$ at room temperature, diluted with $\mathrm{H}_{2} \mathrm{O}$ and extracted with $\mathrm{CH}_{2} \mathrm{Cl}_{2}$. The combined organic extracts were dried over anhydrous $\mathrm{Na}_{2} \mathrm{SO}_{4}$ and concentrated in vacuo. The residue was purified by flash column chromatography on silica gel with an eluent (5:1 to 3:1 hexanes/EtOAc) to give $5(0.18 \mathrm{~g}$, quant.) as a white powder; m.p. $98-100{ }^{\circ} \mathrm{C}$; IR (KBr): $3298,3022,2935,2877$, 2837, 1705, 1604 and $1448 \mathrm{~cm}^{-1}$; ${ }^{1} \mathrm{H}-\mathrm{NMR}\left(270 \mathrm{MHz}, \mathrm{CDCl}_{3}\right) \delta 10.22$ (br s, $1 \mathrm{H}), 7.40(\mathrm{~m}, 5 \mathrm{H}), 6.48(\mathrm{~s}, 2 \mathrm{H}), 5.20(\mathrm{~s}, 2 \mathrm{H}), 3.83(\mathrm{~s}, 3 \mathrm{H}), 2.61(\mathrm{~s}, 3 \mathrm{H}) ;{ }^{13} \mathrm{C}-$ NMR (75 MHz, $\left.\mathrm{CDCl}_{3}\right) \delta 169.9,162.0,158.4,142.7,135.6,128.7,128.3,127.3$, 113.5, 108.9, 97.9, 71.3, 55.3 and 22.0; HRMS (FAB, $m-\mathrm{NBA}$ ): calcd. for $\mathrm{C}_{16} \mathrm{H}_{16} \mathrm{O}_{4}: 272.1049[\mathrm{M}]^{+}$, found: $m / z 272.1049$.

\section{2-Hydroxy-4-methyoxymethoxy-6-methylbenzaldehyde (6)}

To a solution of $5(0.56 \mathrm{~g}, 3.70 \mathrm{mmol})$ in $\mathrm{CH}_{2} \mathrm{Cl}_{2}(37.0 \mathrm{ml}) i-\mathrm{Pr}_{2} \mathrm{NEt}(0.71 \mathrm{ml}$, $4.07 \mathrm{mmol})$ and chloromethyl methyl ether $(\mathrm{MOMCl})(0.56 \mathrm{ml}, 7.39 \mathrm{mmol})$ were added at $0{ }^{\circ} \mathrm{C}$. After stirring for $45 \mathrm{~min}$ at room temperature, the reaction was quenched with $\mathrm{H}_{2} \mathrm{O}$ and extracted with $\mathrm{CH}_{2} \mathrm{Cl}_{2}$. The combined organic extracts were dried over anhydrous $\mathrm{Na}_{2} \mathrm{SO}_{4}$ and concentrated in vacuo. The residue was purified by flash column chromatography on silica gel with an eluent (30:1 hexanes/EtOAc) to give 6 (0.72 g, quant.) as a white powder; m.p. 46-48 ${ }^{\circ} \mathrm{C}$; IR ( $\left.\mathrm{KBr}\right): 3089,2964,1631,1493$ and $1431 \mathrm{~cm}^{-1}$; ${ }^{1} \mathrm{H}-\mathrm{NMR}$ $\left(270 \mathrm{MHz}, \mathrm{CDCl}_{3}\right) \delta 12.31(\mathrm{~s}, 1 \mathrm{H}), 10.11(\mathrm{~s}, 1 \mathrm{H}), 6.42(\mathrm{~d}, J=2.2 \mathrm{~Hz}, 1 \mathrm{H})$, $6.36(\mathrm{~d}, J=2.2 \mathrm{~Hz}, 1 \mathrm{H}), 5.19(\mathrm{~s}, 2 \mathrm{H}), 3.46(\mathrm{~s}, 3 \mathrm{H}), 2.53(\mathrm{~s}, 3 \mathrm{H}) ;{ }^{13} \mathrm{C}-\mathrm{NMR}$ 
a

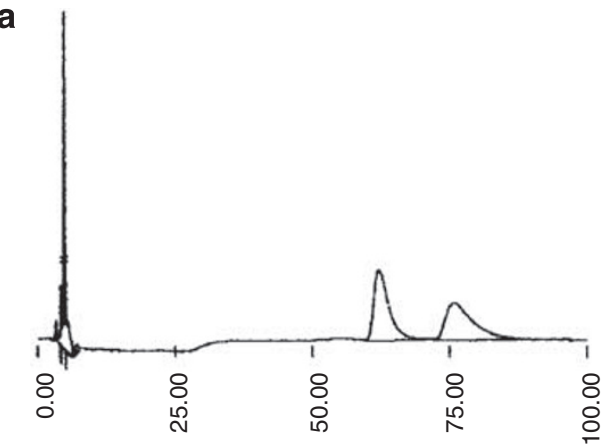

b

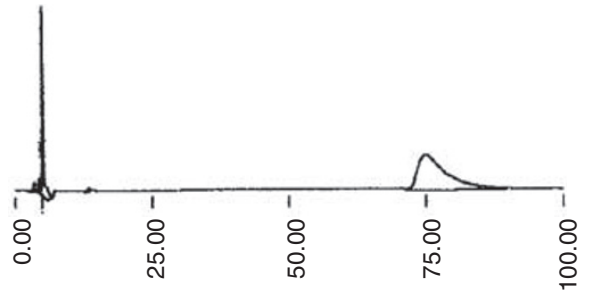

C

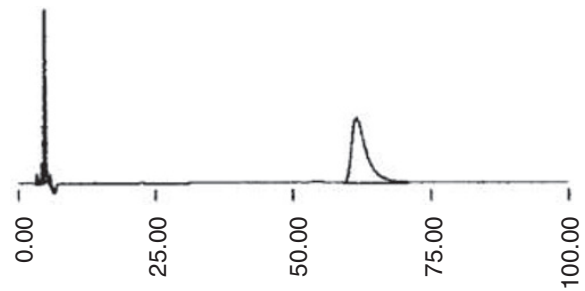

d

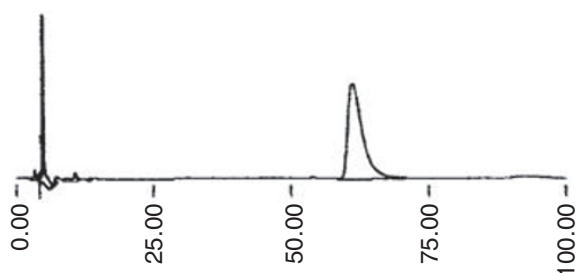

Figure 2 HPLC analysis of natural and synthetic amidepsines B. The abscissa axis indicates retention time. (a) a $1: 1$ mixture of synthetic amidepsines B (+)-1 and (-)-1; (b) synthetic amidepsine B [(+)-1] (derived from D-alanine); (c) synthetic amidepsine B [(-)-1] (derived from L-alanine); (d) natural amidepsine B.

$\left(75 \mathrm{MHz}, \mathrm{CDCl}_{3}\right) \delta 193.1,165.9,164.1,144.0,113.9,110.9,101.3,93.8,56.3$ and 183.3; HRMS (FAB, $m-\mathrm{NBA}$ ): calcd. for $\mathrm{C}_{10} \mathrm{H}_{13} \mathrm{O}_{4}: 197.0814[\mathrm{M}+\mathrm{H}]^{+}$, found: $m / z 197.0808$.

\section{2-Benzyloxy-4-methoxymethoxy-6-methylbenzaldehyde (7)}

To a solution of $6(0.76 \mathrm{~g}, 3.86 \mathrm{mmol})$ in DMF $(19.3 \mathrm{ml}) \mathrm{K}_{2} \mathrm{CO}_{3}(5.33 \mathrm{~g}$, $38.6 \mathrm{mmol})$ and $\mathrm{BnCl}(4.44 \mathrm{ml}, 38.6 \mathrm{mmol})$ were added at $0{ }^{\circ} \mathrm{C}$. After stirring for $45 \mathrm{~min}$ at room temperature, the mixture was warmed to $50^{\circ} \mathrm{C}$ and stirred for $30 \mathrm{~min}$. The mixture was quenched with $\mathrm{H}_{2} \mathrm{O}$ and extracted with $\mathrm{CH}_{2} \mathrm{Cl}_{2}$. The combined organic extracts were dried over anhydrous $\mathrm{Na}_{2} \mathrm{SO}_{4}$ and concentrated in vacuo. The residue was purified by flash column chromatography on silica gel with an eluent (20:1 hexanes/EtOAc) to give 7 (1.09 g, 99\%) as a white powder; m.p. $43-46^{\circ} \mathrm{C}$; IR (KBr): $2927,1674,1601,1450$ and $1415 \mathrm{~cm}^{-1} ;{ }^{1} \mathrm{H}-\mathrm{NMR}\left(270 \mathrm{MHz}, \mathrm{CDCl}_{3}\right) \delta 10.59(\mathrm{~s}, 1 \mathrm{H}), 7.39(\mathrm{~m}, 5 \mathrm{H}), 6.57(\mathrm{~d}$, $J=2.0 \mathrm{~Hz}, 1 \mathrm{H}), 6.48(\mathrm{~d}, J=2.0 \mathrm{~Hz}, 1 \mathrm{H}), 5.19(\mathrm{~s}, 2 \mathrm{H}), 5.13(\mathrm{~s}, 2 \mathrm{H}), 3.48(\mathrm{~s}, 3 \mathrm{H})$, $2.58(\mathrm{~s}, 3 \mathrm{H}) ;{ }^{13} \mathrm{C}-\mathrm{NMR}\left(75 \mathrm{MHz}, \mathrm{CDCl}_{3}\right) \delta 190.6,164.1,161.9,144.5,135.9$, 128.6, 128.1, 127.3, 118.2, 111.3, 98.5, 93.9, 70.5, 56.2 and 22.1; HRMS (FAB, $m-\mathrm{NBA}$ ): calcd. for $\mathrm{C}_{17} \mathrm{H}_{17} \mathrm{O}_{4}: 285.1132[\mathrm{M}-\mathrm{H}]^{-}$, found: $m / z 285.1119$.
2-Benzyloxy-4-methoxymethoxy-6-methylbenzoic acid (8)

To a solution of $7(0.70 \mathrm{~g}, 2.45 \mathrm{mmol})$ in $t-\mathrm{BuOH} / \mathrm{H}_{2} \mathrm{O}(1: 1)(24.4 \mathrm{ml}), 2$ methyl-2-butene $(1.04 \mathrm{ml}, 9.79 \mathrm{mmol}), \mathrm{NaH}_{2} \mathrm{PO}_{4} \cdot 2 \mathrm{H}_{2} \mathrm{O}(1.10 \mathrm{~g}, 7.34 \mathrm{mmol})$ and $\mathrm{NaClO}_{2}(0.66 \mathrm{ml}, 7.34 \mathrm{mmol})$ were added at $0{ }^{\circ} \mathrm{C}$. The reaction mixture was stirred for $24 \mathrm{~h}$ at room temperature, diluted with $\mathrm{H}_{2} \mathrm{O}$ and extracted with $\mathrm{CH}_{2} \mathrm{Cl}_{2}$. The combined organic extracts were dried over anhydrous $\mathrm{Na}_{2} \mathrm{SO}_{4}$ and concentrated in vacuo. The residue was purified by flash column chromatography on silica gel with an eluent $(5: 1$ hexanes/EtOAc) to give $8(0.74 \mathrm{~g}$, quant.) as a yellow oil. IR (KBr): $3168,2927,1697,1601$ and $1448 \mathrm{~cm}^{-1} ;{ }^{1} \mathrm{H}$ NMR $\left(300 \mathrm{MHz}, \mathrm{CDCl}_{3}\right) \delta 9.75($ br s, $1 \mathrm{H}), 7.41(\mathrm{~m}, 5 \mathrm{H}), 6.59(\mathrm{~d}, J=2.2 \mathrm{~Hz}$, $1 \mathrm{H}), 6.58(\mathrm{~d}, J=2.2 \mathrm{~Hz}, 1 \mathrm{H}), 5.17(\mathrm{~s}, 2 \mathrm{H}), 5.16(\mathrm{~s}, 2 \mathrm{H}), 3.47(\mathrm{~s}, 3 \mathrm{H}), 2.51$ $(\mathrm{s}, 3 \mathrm{H}) ;{ }^{13} \mathrm{C}-\mathrm{NMR}\left(75 \mathrm{MHz}, \mathrm{CDCl}_{3}\right) \delta 169.3,159.6,158.3,142.7,135.5,128.7$, $128.3,127.4,114.4,111.5,99.4,94.1,71.4,56.1$ and 21.9; HRMS (FAB, $m$-NBA): calcd. for $\mathrm{C}_{17} \mathrm{H}_{19} \mathrm{O}_{5}: 303.1232[\mathrm{M}+\mathrm{H}]^{+}$, found: $m / z 303.1244$.

2-Benzyloxy-4-methoxymethoxy-6-methylbenzoic acid allyl ester (9) To a solution of $8(1.06 \mathrm{~g}, 3.51 \mathrm{mmol})$ in DMF $(35.1 \mathrm{ml}), \mathrm{NaH}(0.21 \mathrm{~g}$, $5.27 \mathrm{mmol})$ and allyl bromide $(0.91 \mathrm{ml}, 10.5 \mathrm{mmol})$ were added at $0{ }^{\circ} \mathrm{C}$ under $\mathrm{N}_{2}$. After stirring for $30 \mathrm{~min}$, the reaction mixture was warmed to room temperature and stirred for $1.5 \mathrm{~h}$. The mixture was quenched with $\mathrm{H}_{2} \mathrm{O}$ and extracted with EtOAc. The combined organic extracts were dried over anhydrous $\mathrm{Na}_{2} \mathrm{SO}_{4}$ and concentrated in vacuo. The residue was purified by flash column chromatography on silica gel with an eluent (15:1 hexanes/EtOAc) to give 9 (1.14 g, quant.) as a colorless oil. IR (KBr): 2945, 1726, 1599 and $1446 \mathrm{~cm}^{-1}$; ${ }^{1} \mathrm{H}-\mathrm{NMR}\left(270 \mathrm{MHz}, \mathrm{CDCl}_{3}\right) \delta 7.33(\mathrm{~m}, 5 \mathrm{H}), 6.50(\mathrm{~d}, J=2.3 \mathrm{~Hz}$, $2 \mathrm{H}), 5.95(\mathrm{~m}, 1 \mathrm{H}), 5.35(\mathrm{dd}, J=17.2,1.5 \mathrm{~Hz}, 1 \mathrm{H}), 5.19(\mathrm{dd}, J=10.2,1.5 \mathrm{~Hz}$, $1 \mathrm{H}), 5.13(\mathrm{~s}, 2 \mathrm{H}), 5.06(\mathrm{~s}, 2 \mathrm{H}), 4.78(\mathrm{br} \mathrm{d}, J=5.6 \mathrm{~Hz}, 2 \mathrm{H}), 3.44(\mathrm{~s}, 3 \mathrm{H}), 2.30$ (s, $3 \mathrm{H}) ;{ }^{13} \mathrm{C}-\mathrm{NMR}\left(75 \mathrm{MHz}, \mathrm{CDCl}_{3}\right) \delta 167.7,158.8,157.0,138.2,136.5,132.1$, 128.3, 127.7, 127.1, 118.4, 117.8, 109.6, 99.1, 94.2, 70.3, 65.6, 55.9 and 19.7; HRMS (FAB, $m-\mathrm{NBA}$ ): calcd. for $\mathrm{C}_{20} \mathrm{H}_{23} \mathrm{O}_{5}: 343.1545[\mathrm{M}+\mathrm{H}]^{+}$, found: $m / z$ 343.1556.

2-Benzyloxy-4-hydroxy-6-methylbenzoic acid allyl ester (10)

To a solution of $9(2.10 \mathrm{~g}, 6.13 \mathrm{mmol})$ in $\mathrm{CH}_{3} \mathrm{OH}(30.5 \mathrm{ml}), 6 \mathrm{~N} \mathrm{HCl}(30.5 \mathrm{ml})$ was added. After stirring for $30 \mathrm{~min}$ at $60^{\circ} \mathrm{C}$, the reaction mixture was neutralized with a saturated aqueous $\mathrm{NaHCO}_{3}$ solution and extracted with $\mathrm{CH}_{2} \mathrm{Cl}_{2}$. The combined organic extracts were dried over anhydrous $\mathrm{Na}_{2} \mathrm{SO}_{4}$ and concentrated in vacuo. The residue was purified by flash column chromatography on silica gel with an eluent (10:1 hexanes/EtOAc) to give $10(1.83 \mathrm{~g}$, quant.) as a yellow oil. IR (KBr): 3381, 3080, 3033, 2937, 2875, 1697, 1603 and $1458 \mathrm{~cm}^{-1}$; ${ }^{1} \mathrm{H}-\mathrm{NMR}\left(270 \mathrm{MHz}, \mathrm{CDCl}_{3}\right) \delta 7.31(\mathrm{~m}, 5 \mathrm{H}), 6.26(\mathrm{~d}, J=2.0 \mathrm{~Hz}$, $1 \mathrm{H}), 6.20(\mathrm{~d}, J=2.0 \mathrm{~Hz}, 1 \mathrm{H}), 5.93(\mathrm{~m}, 1 \mathrm{H}), 5.35(\mathrm{dd}, J=17.2,1.3 \mathrm{~Hz}, 1 \mathrm{H}), 5.19$ (dd, $J=10.6,1.3 \mathrm{~Hz}, 1 \mathrm{H}), 4.92(\mathrm{~s}, 2 \mathrm{H}), 4.77(\mathrm{br} \mathrm{d}, J=5.6 \mathrm{~Hz}, 2 \mathrm{H}), 2.21(\mathrm{~s}, 3 \mathrm{H})$; ${ }^{13} \mathrm{C}-\mathrm{NMR}\left(75 \mathrm{MHz}, \mathrm{CDCl}_{3}\right) \delta 169.0,158.1,157.5,138.6,136.4,131.8,128.3$, 127.8, 127.1, 118.7, 115.7, 109.6, 98.2, 70.2, 66.0 and 19.6; HRMS (FAB, $m$-NBA): calcd. for $\mathrm{C}_{18} \mathrm{H}_{19} \mathrm{O}_{4}: 299.1283[\mathrm{M}+\mathrm{H}]^{+}$, found: $m / z 299.1296$.

\section{2-Benzyloxy-4-(2-benzyloxy-4-methoxy-6-methyl)} benzoyloxy-6-methylbenzoic acid allyl ester (11)

To a solution of $5(0.19 \mathrm{~g}, 0.69 \mathrm{mmol})$ and $10(0.17 \mathrm{~g}, 0.57 \mathrm{mmol})$ in toluene $(2.3 \mathrm{ml})$, TFAA $(0.57 \mathrm{ml})$ was added. The reaction mixture was stirred for $1 \mathrm{~h}$ at room temperature, quenched with $\mathrm{H}_{2} \mathrm{O}$ and extracted with EtOAc. The combined organic extracts were dried over anhydrous $\mathrm{Na}_{2} \mathrm{SO}_{4}$ and concentrated in vacuo. The residue was purified by flash column chromatography on silica gel with an eluent (40:1 to 20:1 hexanes/EtOAc) to give 11 (0.32 g, quant.) as a white powder; m.p. $122-125^{\circ} \mathrm{C}$; IR (KBr): 2927, 2856, 1738, 1597 and $1437 \mathrm{~cm}^{-1}$; ${ }^{1} \mathrm{H}-\mathrm{NMR}\left(270 \mathrm{MHz}, \mathrm{CDCl}_{3}\right) \delta 7.33(\mathrm{~m}, 10 \mathrm{H}), 6.54(\mathrm{~d}, J=1.7 \mathrm{~Hz}$, $1 \mathrm{H}), 6.52(\mathrm{~d}, J=1.7 \mathrm{~Hz}, 1 \mathrm{H}), 6.45(\mathrm{~d}, J=1.7 \mathrm{~Hz}, 1 \mathrm{H}), 6.40(\mathrm{~d}, J=2.0 \mathrm{~Hz}, 1 \mathrm{H})$, $5.91(\mathrm{~m}, 1 \mathrm{H}), 5.34(\mathrm{dd}, J=17.2,1.2 \mathrm{~Hz}, 1 \mathrm{H}), 5.19(\mathrm{dd}, J=10.2,1.2 \mathrm{~Hz}, 1 \mathrm{H}), 5.09$ (s, 2H), $4.86(\mathrm{~s}, 2 \mathrm{H}), 4.77$ (br d, J=5.6 Hz, 2H), $3.82(\mathrm{~s}, 3 \mathrm{H}), 2.43(\mathrm{~s}, 3 \mathrm{H}), 2.26$ $(\mathrm{s}, 3 \mathrm{H}) ;{ }^{13} \mathrm{C}$-NMR $\left(75 \mathrm{MHz}, \mathrm{CDCl}_{3}\right) \delta 167.3,166.4,161.8,157.8,156.6,152.2$, $139.0,137.9,136.3,136.2,131.9,128.5,128.3,128.1,127.8,127.7,127.3,121.6$, $118.5,115.8,115.5,107.2,104.2,97.4,70.7,70.5,65.8,55.4,20.0$ and 19.4; HRMS (FAB, $m-\mathrm{NBA}+\mathrm{NaI}$ ): calcd. for $\mathrm{C}_{34} \mathrm{H}_{32} \mathrm{O}_{7} \mathrm{Na}$ : $575.2046[\mathrm{M}+\mathrm{Na}]^{+}$, found: $m / z$ 575.2072. 
2-Benzyloxy-4-(2-benzyloxy-4-methoxy-6-methyl) benzoyloxy-6-methylbenzoic acid (12)

To a solution of $11(0.32 \mathrm{~g}, 0.58 \mathrm{mmol})$ in THF $(5.8 \mathrm{ml}), \mathrm{HCOOH}(0.10 \mathrm{ml}$, $2.90 \mathrm{mmol}), \mathrm{Et}_{3} \mathrm{~N}(0.40 \mathrm{ml}, 2.90 \mathrm{mmol})$ and $\mathrm{Pd}\left(\mathrm{PPh}_{3}\right)_{4}(34.0 \mathrm{mg}, 29.0 \mu \mathrm{mol})$ were added at $0{ }^{\circ} \mathrm{C}$. The reaction mixture was stirred for $24 \mathrm{~h}$ at room temperature, quenched with $\mathrm{H}_{2} \mathrm{O}$ and extracted with EtOAc. The combined organic extracts were dried over anhydrous $\mathrm{Na}_{2} \mathrm{SO}_{4}$ and concentrated in vacuo. The residue was purified by flash column chromatography on silica gel with an eluent (1:1 hexanes/EtOAc) to give $\mathbf{1 2}(0.29 \mathrm{~g}, 98 \%)$ as a white powder; m.p. 155-158 ${ }^{\circ} \mathrm{C}$; IR (KBr): 3475, 2991, 2958, 2931, 2873, 2659, 2561, 1741, 1691, 1597 and $1442 \mathrm{~cm}^{-1}$; ${ }^{1} \mathrm{H}-\mathrm{NMR}\left(300 \mathrm{MHz}, \mathrm{CDCl}_{3}\right) \delta 7.36(\mathrm{~m}, 10 \mathrm{H}), 6.62$ $(\mathrm{d}, J=1.8 \mathrm{~Hz}, 1 \mathrm{H}), 6.61(\mathrm{~d}, 1 \mathrm{H}, J=1.8 \mathrm{~Hz}), 6.47(\mathrm{~d}, 1 \mathrm{H}, J=1.9 \mathrm{~Hz}), 6.43$ (d, $J=2.1 \mathrm{~Hz}, 1 \mathrm{H}), 5.10(\mathrm{~s}, 2 \mathrm{H}), 4.93(\mathrm{~s}, 2 \mathrm{H}), 3.83(\mathrm{~s}, 3 \mathrm{H}), 2.45(\mathrm{~s}, 3 \mathrm{H}), 2.43$ $(\mathrm{s}, 3 \mathrm{H}) ;{ }^{13} \mathrm{C}-\mathrm{NMR}\left(75 \mathrm{MHz}, \mathrm{CDCl}_{3}\right) \delta 170.1,166.3,161.9,157.9,157.3,152.9$, 140.7, 139.2, 136.3, 135.5, 128.6, 128.5, 128.2, 128.2, 127.7, 127.4, 119.2, 117.0, $115.3,107.3,104.7,97.4,71.2,70.7,55.4,20.8$ and 20.1; HRMS (FAB, $m-$ $\mathrm{NBA}+\mathrm{NaI}$ ): calcd. for $\mathrm{C}_{31} \mathrm{H}_{28} \mathrm{O}_{7} \mathrm{Na}: 535.1733[\mathrm{M}+\mathrm{Na}]^{+}$, found: $\mathrm{m} / z 535.1758$.

\section{2-Benzyloxy-4-(2-benzyloxy-4-methoxy-6-methyl) benzoyloxy-6-methylbenzoyl fluoride (13)}

To a solution of $12(0.03 \mathrm{~g}, 0.06 \mathrm{mmol})$ in $\mathrm{Et}_{2} \mathrm{O} / \mathrm{CH}_{2} \mathrm{Cl}_{2}(2: 1)(0.9 \mathrm{ml})$ DAST $(18.6 \mu \mathrm{l}, 0.14 \mathrm{mmol})$ was added. The reaction mixture was stirred for $1.5 \mathrm{~h}$ at room temperature, quenched with $\mathrm{H}_{2} \mathrm{O}$ and extracted with $\mathrm{CH}_{2} \mathrm{Cl}_{2}$. The combined organic extracts were dried over anhydrous $\mathrm{Na}_{2} \mathrm{SO}_{4}$ and concentrated in vacuo. The residue was purified by flash column chromatography on silica gel with an eluent (25:1 hexanes/EtOAc) to give $\mathbf{1 3}(27.0 \mathrm{mg}, 89 \%)$ as a white powder; m.p. $111-114^{\circ} \mathrm{C}$; IR (KBr): $3624,3458,3035,2924,2852,1817$, 1732, 1593 and $1442 \mathrm{~cm}^{-1}$; ${ }^{1} \mathrm{H}-\mathrm{NMR}\left(300 \mathrm{MHz}, \mathrm{CDCl}_{3}\right) \delta 7.38(\mathrm{~m}, 10 \mathrm{H}), 6.62$ (s, $1 \mathrm{H}), 6.48(\mathrm{~d}, J=2.0 \mathrm{~Hz}, 1 \mathrm{H}), 6.44(\mathrm{~d}, J=1.9 \mathrm{~Hz}, 1 \mathrm{H}), 5.10(\mathrm{~s}, 2 \mathrm{H}), 4.92$ $(\mathrm{s}, 2 \mathrm{H}), 3.84(\mathrm{~s}, 3 \mathrm{H}), 2.46(\mathrm{~s}, 3 \mathrm{H}), 2.41(\mathrm{~s}, 3 \mathrm{H}) ;{ }^{13} \mathrm{C}-\mathrm{NMR}\left(75 \mathrm{MHz}, \mathrm{CDCl}_{3}\right) \delta$ 166.0, 162.0, $158.9\left({ }^{3} J_{\mathrm{CF}}=2.2 \mathrm{~Hz}\right), 158.0,156.6\left({ }^{1} J_{\mathrm{CF}}=351.7 \mathrm{~Hz}\right), 154.5,141.8$ $\left({ }^{3} J_{\mathrm{CF}}=2.4 \mathrm{~Hz}\right), 139.2,136.2,135.6,128.5,128.2,128.0,127.7,127.1,116.6$ $\left({ }^{4} J_{\mathrm{CF}}=2.4 \mathrm{~Hz}\right), 115.0,114.5\left({ }^{2} J_{\mathrm{CF}}=56.2 \mathrm{~Hz}\right), 107.3,104.7,97.4,70.7,70.6,55.3$, $20.5\left({ }^{4} J_{\mathrm{CF}}=1.2 \mathrm{~Hz}\right), 20.0$; HR-MS (FAB, $\left.m-\mathrm{NBA}+\mathrm{NaI}\right)$ : calcd. for $\mathrm{C}_{31} \mathrm{H}_{27} \mathrm{FO}_{6-}$ Na: $537.1684[\mathrm{M}+\mathrm{Na}]^{+}$, found: $m / z 537.1687$.

\section{2-Benzyloxy-4-[2-benzyloxy-4-(2-benzyloxy-4-methoxy-6-methyl)} benzoyloxy-6-methyl] benzoyloxy-6-methylbenzoic acid allyl ester (14) To a solution of $10(22.0 \mathrm{mg}, 0.07 \mathrm{mmol})$ in THF $(0.5 \mathrm{ml}), \mathrm{NaH}(6.0 \mathrm{mg}$, $0.15 \mathrm{mmol}$ ) was added at $0{ }^{\circ} \mathrm{C}$ under $\mathrm{N}_{2}$. After stirring for $5 \mathrm{~min}$ at room temperature, a solution of $\mathbf{1 3}(26.0 \mathrm{mg}, 0.05 \mathrm{mmol})$ in THF $(0.5 \mathrm{ml})$ was added dropwise. After $30 \mathrm{~min}$, the mixture was treated with $\mathrm{MOMCl}(3.8 \mu \mathrm{l}$, $0.05 \mathrm{mmol}$ ) for the conversion of an excess amount of $\mathbf{1 0}$ into 9 and then stirred for $30 \mathrm{~min}$. The resulting mixture was quenched with $\mathrm{H}_{2} \mathrm{O}$ and extracted with EtOAc. The combined organic extracts were dried over anhydrous $\mathrm{Na}_{2} \mathrm{SO}_{4}$ and concentrated in vacuo. The residue was purified by flash column chromatography on silica gel with an eluent (10:1 to $1: 1$ hexanes/EtOAc) to give $\mathbf{1 4}$ ( $40.0 \mathrm{mg}$, quant.) as a white powder; m.p. $183-187^{\circ} \mathrm{C}$; IR ( $\mathrm{KBr}$ ): 2925,2858 , 1741,1597 and $1439 \mathrm{~cm}^{-1} ;{ }^{1} \mathrm{H}-\mathrm{NMR}\left(270 \mathrm{MHz}, \mathrm{CDCl}_{3}\right) \delta 7.33(\mathrm{~m}, 15 \mathrm{H}), 6.59$ $(\mathrm{d}, J=2.0 \mathrm{~Hz}, 1 \mathrm{H}), 6.58(\mathrm{~d}, J=2.0 \mathrm{~Hz}, 1 \mathrm{H}), 6.49(\mathrm{~d}, J=2.1 \mathrm{~Hz}, 1 \mathrm{H}), 6.47(\mathrm{~d}$, $J=2.0 \mathrm{~Hz}, 1 \mathrm{H}), 6.46(\mathrm{~d}, J=2.1 \mathrm{~Hz}, 1 \mathrm{H}), 6.41(\mathrm{~d}, J=2.0 \mathrm{~Hz}, 1 \mathrm{H}), 5.91(\mathrm{~m}, 1 \mathrm{H})$, 5.33 (dd, $J=17.2,1.5 \mathrm{~Hz}, 1 \mathrm{H}), 5.18$ (dd, $J=10.5,1.5 \mathrm{~Hz}, 1 \mathrm{H}), 5.10(\mathrm{~s}, 2 \mathrm{H}), 4.85$ (s, $2 \mathrm{H}), 4.82(\mathrm{~s}, 2 \mathrm{H}), 4.76(\mathrm{br} \mathrm{d}, J=5.9 \mathrm{~Hz}, 2 \mathrm{H}), 3.83(\mathrm{~s}, 3 \mathrm{H}), 2.45(\mathrm{~s}, 3 \mathrm{H}), 2.37$ $(\mathrm{s}, 3 \mathrm{H}), 2.24(\mathrm{~s}, 3 \mathrm{H}) ;{ }^{13} \mathrm{C}-\mathrm{NMR}\left(75 \mathrm{MHz}, \mathrm{CDCl}_{3}\right) \delta 167.3,166.4,166.0,161.9$, $157.9,157.0,156.6,152.8,152.0,139.1,138.2,137.9,136.3,136.2,136.0,132.0$, $128.5,128.5,128.4,128.2,128.2,127.9,127.9,127.8,127.3,121.8,120.5,118.6$, $116.0,115.7,115.4,107.2,104.1,97.4,70.8,70.7,70.5,65.8,55.4,20.1,19.4$ and 19.3; HRMS (FAB, $m-\mathrm{NBA}+\mathrm{NaI}$ ): calcd. for $\mathrm{C}_{49} \mathrm{H}_{44} \mathrm{O}_{10} \mathrm{Na}: 815.2832[\mathrm{M}+\mathrm{Na}]^{+}$, found: $m / z$ 815.2806.

\section{2-Benzyloxy-4-[2-benzyloxy-4-(2-benzyloxy-4-methoxy-6-} methyl)benzoyloxy-6-methyl] benzoyloxy-6-methylbenzoic acid (15) To a solution of $\mathbf{1 4}(40.0 \mathrm{mg}, 0.05 \mathrm{mmol})$ in THF $(0.5 \mathrm{ml}), \mathrm{HCOOH}(9.4 \mu \mathrm{l}$, $0.25 \mathrm{mmol}), \mathrm{Et}_{3} \mathrm{~N}(34.8 \mu \mathrm{l}, 0.25 \mathrm{mmol})$ and $\mathrm{Pd}\left(\mathrm{PPh}_{3}\right)_{4}(29.0 \mathrm{mg}, 3.00 \mu \mathrm{mol})$ were added at $0{ }^{\circ} \mathrm{C}$. The reaction mixture was stirred for $24 \mathrm{~h}$ at room temperature, quenched with $\mathrm{H}_{2} \mathrm{O}$ and extracted with EtOAc. The combined organic extracts were dried over anhydrous $\mathrm{Na}_{2} \mathrm{SO}_{4}$ and concentrated in vacuo. The residue was purified by flash column chromatography on silica gel with an eluent (2:1 hexanes/EtOAc) to give $\mathbf{1 5}(38.0 \mathrm{mg}$, quant.) as a white powder; m.p. $215-216^{\circ} \mathrm{C}$; IR (KBr): 3446, 2924, 2858, 1745, 1691, 1597 and $1441 \mathrm{~cm}^{-1}$; ${ }^{1} \mathrm{H}-\mathrm{NMR}\left(270 \mathrm{MHz}, \mathrm{CDCl}_{3}\right) \delta 7.40(\mathrm{~m}, 15 \mathrm{H}), 6.60($ br s, $2 \mathrm{H}), 6.58$ $(\mathrm{d}, J=2.0 \mathrm{~Hz}, 1 \mathrm{H}), 6.56(\mathrm{~d}, J=2.1 \mathrm{~Hz}, 1 \mathrm{H}), 6.47(\mathrm{~d}, J=2.2 \mathrm{~Hz}, 1 \mathrm{H}), 6.42$ (d, $J=2.3 \mathrm{~Hz}, 1 \mathrm{H}), 5.10(\mathrm{~s}, 2 \mathrm{H}), 4.88(\mathrm{~s}, 2 \mathrm{H}), 4.85(\mathrm{~s}, 2 \mathrm{H}), 3.83(\mathrm{~s}, 3 \mathrm{H}), 2.47$ $(\mathrm{s}, 3 \mathrm{H}), 2.44(\mathrm{~s}, 3 \mathrm{H}), 2.38(\mathrm{~s}, 3 \mathrm{H}) ;{ }^{13} \mathrm{C}-\mathrm{NMR}\left(75 \mathrm{MHz}, \mathrm{CDCl}_{3}\right) \delta 167.2,166.4$, 165.9, 161.9, 158.0, 157.5, 157.2, 153.0, 152.9, 142.4, 139.2, 138.4, 136.3, 136.0, $135.1,128.8,128.6,128.6,128.5,128.3,128.2,128.0,127.9,127.7,120.2,118.4$, 117.6, 116.1, 115.4, 107.3, 104.6, 104.2, 97.5, 71.6, 70.9, 70.8, 55.5, 21.5, 20.1 and 19.5; HRMS (FAB, $m-\mathrm{NBA}+\mathrm{NaI}$ ): calcd. for $\mathrm{C}_{46} \mathrm{H}_{40} \mathrm{O}_{10} \mathrm{Na}$ : 775.2519 $[\mathrm{M}+\mathrm{Na}]^{+}$, found: $m / z 775.2510$.

\section{(2S)-2\{2-Benzyloxy-4-[2-benzyloxy-4-(2-benzyloxy-4-methoxy-6-} methyl)benzoyloxy-6-methyl]benzoyloxy-6methylbenzoy\}aminopropionic acid benzyl ester $[(+)-(16)]$

To a solution of $15(92.0 \mathrm{mg}, 0.12 \mathrm{mmol})$ in DMF $(1.2 \mathrm{ml}), \mathrm{L}-\mathrm{Ala}-\mathrm{OBn} \cdot \mathrm{HCl}$ $(58.0 \mathrm{mg}, \quad 0.27 \mathrm{mmol}), \mathrm{Et}_{3} \mathrm{~N} \quad(68.0 \mu \mathrm{l}, 0.49 \mathrm{mmol})$ and $\mathrm{BOP}(135.0 \mathrm{mg}$, $0.30 \mathrm{mmol}$ ) were added. The reaction mixture was stirred for $3.5 \mathrm{~h}$ at room temperature, quenched with $\mathrm{H}_{2} \mathrm{O}$ and extracted with EtOAc. The combined organic extracts were dried over anhydrous $\mathrm{Na}_{2} \mathrm{SO}_{4}$ and concentrated in vacuo. The residue was purified by flash column chromatography on silica gel with an eluent (3:1 to 2:1 hexanes/EtOAc) to give (+)-(16) $(88.0 \mathrm{mg}, 79 \%)$ as a white powder; m.p. $179-181^{\circ} \mathrm{C}$; $[\alpha]^{27}{ }_{\mathrm{D}}+21.5\left(\mathrm{c} 0.1, \mathrm{CHCl}_{3}\right)$; IR $(\mathrm{KBr}): 2925,1741$, 1595 and $1446 \mathrm{~cm}^{-1}$; ${ }^{1} \mathrm{H}-\mathrm{NMR}\left(270 \mathrm{MHz}, \mathrm{CDCl}_{3}\right) \delta 7.38(\mathrm{~m}, 20 \mathrm{H}), 6.61$ (br s, $2 \mathrm{H}), 6.51(\mathrm{~d}, J=1.3 \mathrm{~Hz}, 1 \mathrm{H}), 6.48(\mathrm{br} \mathrm{s}, 2 \mathrm{H}), 6.43(\mathrm{~d}, J=1.6 \mathrm{~Hz}, 1 \mathrm{H}), 6.41(\mathrm{~d}$, $J=7.6 \mathrm{~Hz}, 1 \mathrm{H}), 5.18(\mathrm{~d}, J=12.3 \mathrm{~Hz}, 1 \mathrm{H}), 5.13(\mathrm{~d}, J=12.3 \mathrm{~Hz}, 1 \mathrm{H}), 5.11$ (s, 2H), $4.87(\mathrm{~s}, 2 \mathrm{H}), 4.79(\mathrm{~m}, 1 \mathrm{H}), 4.78(\mathrm{~s}, 2 \mathrm{H}), 3.84(\mathrm{~s}, 3 \mathrm{H}), 2.47(\mathrm{~s}, 3 \mathrm{H})$, $2.39(\mathrm{~s}, 3 \mathrm{H}), 2.28(\mathrm{~s}, 3 \mathrm{H}), 1.35(\mathrm{~d}, J=7.0 \mathrm{~Hz}, 3 \mathrm{H}) ;{ }^{13} \mathrm{C}-\mathrm{NMR}\left(75 \mathrm{MHz}, \mathrm{CDCl}_{3}\right) \delta$ $172.5,166.4,166.4,166.2,161.9,157.9,157.1,156.2,152.8,151.8,139.1,139.1$, $138.3,136.3,136.0,136.0,135.3,128.6,128.6,128.6,128.4,128.4,128.3,128.2$, $128.2,128.0,128.0,127.8,127.6,123.8,120.5,116.2,116.0,115.4,107.3,104.2$, 104.0, 97.4, 70.8, 70.8, 70.5, 67.0, 55.4, 48.1, 20.1, 19.5 and 18.3; HRMS (FAB, $m$-NBA): calcd. for $\mathrm{C}_{56} \mathrm{H}_{52} \mathrm{NO}_{11}: 914.3540[\mathrm{M}+\mathrm{H}]^{+}$, found: $\mathrm{m} / z$ 914.3497.

\section{(2S)-2\{2-Benzyloxy-4-[2-benzyloxy-4-(2-benzyloxy-4-methoxy-6-} methyl)benzoyloxy-6-methyl]benzoyloxy-6methylbenzoy $\}$ aminopropionic acid $\{(-)$-Amidepsine B $[(-)-1]\}$ A mixture of $(+)-(16)(25.1 \mathrm{mg}, 0.03 \mathrm{mmol})$ and $\mathrm{Pd}(\mathrm{OH})_{2}(12.2 \mathrm{mg})$ in THF/EtOH (1:1) $(2.8 \mathrm{ml})$ was stirred under $\mathrm{H}_{2}$ atmosphere for $16 \mathrm{~h}$. The mixture was filtered through a pad of Celite and the filtrate was concentrated. The residue was purified by preparative TLC on silica gel $\left(5: 1 \mathrm{CHCl}_{3} / \mathrm{CH}_{3} \mathrm{OH}\right)$ with an eluent $\left(3: 1 \mathrm{CHCl}_{3} / \mathrm{CH}_{3} \mathrm{OH}\right)$ to give (-)-(1) as a white powder $(13.0 \mathrm{mg}$, $87 \%)$. Moreover, this sample was dissolved in $\mathrm{CHCl}_{3} / \mathrm{CH}_{3} \mathrm{OH}(25: 1)(5 \mathrm{ml})$ and washed with $1 \%$ aqueous $\mathrm{H}_{3} \mathrm{PO}_{4}(1 \mathrm{ml})$. The organic phase was dried over anhydrous $\mathrm{Na}_{2} \mathrm{SO}_{4}$ and concentrated in vacuo to give (-)-(1) (12.4 mg, $\left.83 \%\right)$ as a white powder; m.p. $153-156{ }^{\circ} \mathrm{C}$; $[\alpha]^{29} \mathrm{D}-17.3$ (c 0.1, $\left.\mathrm{CH}_{3} \mathrm{OH}\right)$; IR (KBr): 3087, 2937, 2858, 1668, 1610 and $1454 \mathrm{~cm}^{-1}$; ${ }^{1} \mathrm{H}-\mathrm{NMR}\left(270 \mathrm{MHz}, \mathrm{CDCl}_{3}\right) \delta 10.4(\mathrm{br}$ $\mathrm{s}, 1 \mathrm{H}), 8.44(\mathrm{~d}, J=6.8 \mathrm{~Hz}, 1 \mathrm{H}), 6.68(\mathrm{br} \mathrm{s}, 1 \mathrm{H}), 6.66(\mathrm{br} \mathrm{s}, 1 \mathrm{H}), 6.56(\mathrm{br} \mathrm{s}, 1 \mathrm{H})$, $6.53(\mathrm{br} \mathrm{s}, 1 \mathrm{H}), 6.39(\mathrm{br} \mathrm{s}, 1 \mathrm{H}), 6.36(\mathrm{br} \mathrm{s}, 1 \mathrm{H}), 4.35(\mathrm{~m}, 1 \mathrm{H}), 3.74(\mathrm{~s}, 3 \mathrm{H}), 2.45$ (s, 3H), $2.38(\mathrm{~s}, 3 \mathrm{H}), 2.35(\mathrm{~s}, 3 \mathrm{H}), 1.31(\mathrm{~d}, J=7.1 \mathrm{~Hz}, 3 \mathrm{H}) ;{ }^{13} \mathrm{C}-\mathrm{NMR}(75 \mathrm{MHz}$, $\left.\mathrm{CDCl}_{3}\right) \delta 174.1,166.7,166.4,165.8,162.1,159.1,156.1,155.0,152.0,150.5$, 139.6, 137.8, 137.6, 123.5, 118.4, 114.1, 113.3, 110.7, 108.1, 107.1, 106.4, 99.0, 55.2, 47.6, 20.8, 19.2, 18.8 and 16.9; HRMS (FAB, $m-\mathrm{NBA}$ ): calcd. for $\mathrm{C}_{28} \mathrm{H}_{26} \mathrm{NO}_{11}: 552.1506[\mathrm{M}-\mathrm{H}]^{-}$, found: $m / z$ 552.1510.

(2R)-2\{2-Benzyloxy-4-[2-benzyloxy-4-(2-benzyloxy-4-methoxy-6methyl)benzoyloxy-6-methyl]benzoyloxy-6methylbenzoy\}aminopropionic acid benzyl ester [(-)-(16)]

According to the conversion of $\mathbf{1 5}$ into (+)-16, $15(30.0 \mathrm{mg}, 0.04 \mathrm{mmol})$ was subjected to a condensation reaction with $\mathrm{D}-\mathrm{Ala}-\mathrm{OBn} \cdot \mathrm{p}-\mathrm{TsOH}(21.8 \mathrm{mg}$, $0.060 \mathrm{mmol})$ to afford $(-)-(\mathbf{1 6})(32.6 \mathrm{mg}, 89 \%)$ as a white powder; m.p. $181-183^{\circ} \mathrm{C} ;[\alpha]^{23} \mathrm{D}-8.7\left(c \mathrm{0}, 1, \mathrm{CHCl}_{3}\right)$; IR (KBr): 2925, 1741, 1595 and 
$1446 \mathrm{~cm}^{-1} ;{ }^{1} \mathrm{H}-\mathrm{NMR}\left(270 \mathrm{MHz}, \mathrm{CDCl}_{3}\right) \delta 7.38(\mathrm{~m}, 20 \mathrm{H}), 6.61$ (br s, 2H), 6.51 (d, $J=1.3 \mathrm{~Hz}, 1 \mathrm{H}), 6.48(\mathrm{br} \mathrm{s}, 2 \mathrm{H}), 6.43(\mathrm{~d}, J=1.6 \mathrm{~Hz}, 1 \mathrm{H}), 6.41(\mathrm{~d}, J=7.6 \mathrm{~Hz}$, $1 \mathrm{H}), 5.18(\mathrm{~d}, J=12.3 \mathrm{~Hz}, 1 \mathrm{H}), 5.13(\mathrm{~d}, J=12.3 \mathrm{~Hz}, 1 \mathrm{H}), 5.11(\mathrm{~s}, 2 \mathrm{H}), 4.87(\mathrm{~s}$, $2 \mathrm{H}), 4.79(\mathrm{~m}, 1 \mathrm{H}), 4.78(\mathrm{~s}, 2 \mathrm{H}), 3.84(\mathrm{~s}, 3 \mathrm{H}), 2.47(\mathrm{~s}, 3 \mathrm{H}), 2.39(\mathrm{~s}, 3 \mathrm{H}), 2.28(\mathrm{~s}$, $3 \mathrm{H}), 1.35(\mathrm{~d}, J=7.0 \mathrm{~Hz}, 3 \mathrm{H}) ;{ }^{13} \mathrm{C}-\mathrm{NMR}\left(75 \mathrm{MHz}, \mathrm{CDCl}_{3}\right) \delta 172.5,166.4,166.4$, $166.2,161.9,157.9,157.1,156.2,152.8,151.8,139.1,139.1,138.3,136.3,136.0$, $136.0,135.3,128.6,128.6,128.6,128.4,128.4,128.3,128.2,128.2,128.0,128.0$, $127.8,127.6,123.8,120.5,116.2,116.0,115.4,107.3,104.2,104.0,97.4,70.8$, $70.8, \quad 70.5, \quad 67.0, \quad 55.4, \quad 48.1, \quad 20.1, \quad 19.5$ and 18.3; HRMS (FAB, $m-\mathrm{NBA}+\mathrm{NaI})$ : calcd. for $\mathrm{C}_{56} \mathrm{H}_{51} \mathrm{NO}_{11} \mathrm{Na}$ : $936.3360[\mathrm{M}+\mathrm{Na}]^{+}$, found: $m / z$ 936.3383.

(2R)-2\{2-Benzyloxy-4-[2-benzyloxy-4-(2-benzyloxy-4-methoxy-6methyl)benzoyloxy-6-methyl] benzoyloxy-6methylbenzoy $\}$ aminopropionic acid $\{(+)$-Amidepsine $\mathbf{B}[(+)-1]\}$ According to the conversion of (+)-16 into (-)-1, (-)-(16) $(30.8 \mathrm{mg}$, $0.03 \mathrm{mmol})$ gave $(+)-(\mathbf{1})(17.7 \mathrm{mg}, 94 \%)$ as a white powder; m.p. $156-159^{\circ} \mathrm{C}$; $[\alpha]^{25} \mathrm{D}+10.4\left(c\right.$ 0.1, $\left.\mathrm{CH}_{3} \mathrm{OH}\right) ; \mathrm{IR}(\mathrm{KBr}): 3087,2937,2858,1668,1610$ and $1454 \mathrm{~cm}^{-1} ;{ }^{1} \mathrm{H}-\mathrm{NMR}\left(270 \mathrm{MHz}, \mathrm{CDCl}_{3}\right) \delta 10.4$ (br s, $\left.1 \mathrm{H}\right), 8.44$ $(\mathrm{d}, J=6.8 \mathrm{~Hz}, 1 \mathrm{H}), 6.68$ (br s, $1 \mathrm{H}), 6.66($ br s, $1 \mathrm{H}), 6.56$ (br s, $1 \mathrm{H})$, 6.53 (br s, 1H), 6.39 (br s, $1 \mathrm{H}), 6.36($ br s, $1 \mathrm{H}), 4.35(\mathrm{~m}, 1 \mathrm{H}), 3.74$ (s, 3H), $2.45(\mathrm{~s}, 3 \mathrm{H}), 2.38(\mathrm{~s}, 3 \mathrm{H}), 2.35(\mathrm{~s}, 3 \mathrm{H}), 1.31(\mathrm{~d}, J=7.1 \mathrm{~Hz}, 3 \mathrm{H})$;
${ }^{13} \mathrm{C}-\mathrm{NMR} \quad\left(75 \mathrm{MHz}, \mathrm{CDCl}_{3}\right) \quad \delta 174.1,166.7,166.4,165.8,162.1,159.1$, $156.1, \quad 155.0, \quad 152.0, \quad 150.5, \quad 139.6, \quad 137.8, \quad 137.6, \quad 123.5, \quad 118.4, \quad 114.1$, 113.3, 110.7, 108.1, 107.1, 106.4, 99.0, 55.2, 47.6, 20.8, 19.2, 18.8 and 16.9; HRMS (FAB, $m-\mathrm{NBA}$ ): calcd. for $\mathrm{C}_{28} \mathrm{H}_{28} \mathrm{NO}_{11}: 554.1662[\mathrm{M}+\mathrm{H}]^{+}$, found: $m / z$ 554.1663.

1 Tomoda, H., Ito, M., Tabata, N., Masuma, R., Yamaguchi, Y. \& Ōmura, S. Amidepsines, inhibitors of diacylglycerol acyltransferase produced by Humicola sp. FO-2942. I. Production, isolation and biological properties. J. Antibiot. 48, 937-941 (1995).

2 Tomoda, H., Tabata, N., Ito, M. \& Ōmura, S. Amidepsines, inhibitors of diacylglycerol acyltransferase produced by Humicola sp. FO-2942. II. Structure elucidation of amidepsines A, B and C. J. Antibiot. 48, 942-947 (1995).

3 Tomoda, H., Yamaguchi, Y., Tabata, N., Kobayashi, T., Masuma, R., Tanaka, H. \& Ōmura, S. Amidepsine E, an inhibitor of diacylglycerol acyltransferase produced by Humicola sp. F0-5969. J. Antibiot. 49, 929-931 (1996).

4 Koch, K., Podlech, J., Pfeiffer, E. \& Metzler, M. Total synthesis of alternariol. J. Org. Chem. 70, 3275-3276 (2005).

5 Katoh, T., Ohmori, O., Iwasaki, K. \& Inoue, M. Synthetic studies on Sch 202596, an antagonist of the galanin receptor subtype GalR1: an efficient synthesis of ( \pm )-geodin, the spirocoumaranone part of Sch 202596. Tetrahedron 58, 1289-1299 (2002).

6 Nicolaou, K. C., Mitchell, H. J., Suzuki, H., Rodríguez, R. M., Baudoin, O. \& Fylaktakidou, K. C. Total synthesis of everninomicin 13,384-1 Part 1: Synthesis of the $A_{1} B(A) C$ fragment. Angew Chem. Int. Ed. 38, 3334-3339 (1999). 